Research Article

\title{
Automatic Jamming Modulation Classification Exploiting Convolutional Neural Network for Cognitive Radar
}

\author{
Feng Wang $\mathbb{D}^{1}{ }^{1}$ Shanshan Huang, ${ }^{1}$ and Chao Liang ${ }^{2}$ \\ ${ }^{1}$ College of Computer and Information, Hohai University, West Focheng Road No. 8, Jiangning District, Nanjing 211100, China \\ ${ }^{2}$ Science and Technology on Electronic Information Control Laboratory, \\ Southwest China Research Institute of Electronic Equipment, Chengdu, Sichuan, China
}

Correspondence should be addressed to Feng Wang; jihonghope@aliyun.com

Received 24 April 2020; Revised 8 August 2020; Accepted 12 August 2020; Published 21 September 2020

Academic Editor: Luigi Rodino

Copyright (C) 2020 Feng Wang et al. This is an open access article distributed under the Creative Commons Attribution License, which permits unrestricted use, distribution, and reproduction in any medium, provided the original work is properly cited.

\begin{abstract}
Sensing the external complex electromagnetic environment is an important function for cognitive radar, and the concept of cognition has attracted wide attention in the field of radar since it was proposed. In this paper, a novel method based on an idea of multidimensional feature map and convolutional neural network (CNN) is proposed to realize the automatic modulation classification of jamming entering the cognitive radar system. The multidimensional feature map consists of two envelope maps before and after the pulse compression processing and a time-frequency map of the receiving beam signal. Drawing the onedimensional envelope in a 2-dimensional plane and quantizing the time-frequency data to a 2-dimensional plane, we treat the combination of the three planes (multidimensional feature map) as one picture. A CNN-based algorithm with linear kernel sensing the three planes simultaneously is selected to accomplish jamming classification. The classification of jamming, such as noise frequency modulation jamming, noise amplitude modulation jamming, slice jamming, and dense repeat jamming, is validated by computer simulation. A performance comparison study on convolutional kernels in different size demonstrates the advantage of selecting the linear kernel.
\end{abstract}

\section{Introduction}

Cognitive radar proposed in [1] senses the external electromagnetic environment by recognizing the external emitters and accomplishes antijamming, clutter suppression, and target detection based on the environment information and prior knowledge. Hence, it has significant potential for the optimization and improvement of the performance of the radar. Radar for military applications usually faces a complex electromagnetic environment, especially jamming emitted from electronic countermeasure systems, which must be rejected before target detection. Jamming environment perception is an essential part of the antijamming technology [2-4] for cognitive radar, and it is the premise and foundation on how to employ the various antijamming measures. For example, frequency agility [5] requiring jamming power measurement on each working frequency point can be regarded as a type of simple environment-cognition waveform transmission technology. The perception of jamming refers to the acquirement of the following parameters and status of the interferences: jamming number, azimuth, modulation type, power, frequency bandwidth, and so on. The direction of arrival and number estimation of the interferences have been discussed widely in the field of array signal processing [6-8]. For instance, multiple signal classification (MUSIC) algorithm [9] is usually used to measure the direction of the interferences, and the Gerschgorin disk algorithm [10] is employed to estimate the number of the interferences. Measurement of the jamming power and working frequency bandwidth is widely in use in modern radar systems. However, few papers are devoted to classifying the modulation classes of the radar jamming.

Different antijamming techniques are suitable for different jamming modulation mode. Therefore, in order to make the selection of the antijamming measures more 
intelligent for cognitive radar, it is essential to recognize the modulation class of different types of interferences. We give an explanation of the antijamming process in a cognitive radar in Figure 1. The antijamming techniques including sidelobe cancellation (SLC), sidelobe blanking (SLB), constant false alarm rate (CFAR) processing, and antijamming transmitting waveform are controlled by the results of jamming sensing. The perception of jamming is implemented after digital beamforming (DBF) or pulse compression of the array data. In this example, the sensing of jamming is composed of four parts: (1) jamming power measurement using DBF; (2) jamming bandwidth measurement using fast Fourier transform (FFT); (3) direction of arrival estimation using MUSIC; (4) automatic modulation classification of jamming (AMCOJ). We focus on the automatic modulation classification of jamming in this paper.

The classes of jamming modulation can be divided into noise frequency modulation jamming [11], repeater jamming [12], pulse jamming [13], frequency shift jamming [14], and so on. Since there are few studies on AMCOJ in the field of radar, the automatic modulation classification (AMC) of communication and radar signals in the related areas can be used as references.

In the related fields of communication and electronic warfare, there are a great number of studies on automatic modulation classification of communication signals and radar signals. AMC is regarded as an issue of pattern recognition with two processing stages. Firstly, the features of the signals are extracted by various processing algorithms, such as cumulants [15], radar ambiguity function [16], and time-frequency analysis [17]. Secondly, the features are used as the input of certain classification algorithms, such as support vector machine [18] and fuzzy clustering [19]. Recently, some papers propose approaches based on deep learning accomplishing feature extraction and modulation classification simultaneously. The study in [20] adopts the method of heterogeneous deep model fusion, combining convolutional neural network (CNN), and long short-term memory and realizes AMC of multiple single carriermodulated signals. An algorithm based on deep learning self-coding network is introduced in [21], which achieves AMC of five classes of signals: binary phase shift keying (BPSK), 8-phase shift keying (8PSK), 4-quadrature amplitude modulation (4QAM), 16-quadrature amplitude modulation (16QAM), and 64-quadrature amplitude modulation (64QAM). Compared with the traditional sparse autoencoder, the nonnegative constrained autoencoder improves the sparsity and classification accuracy. In [22], a k-sparse autoencoder-based classifier with characteristics of disordered input data called disordered deep neural network is proposed, reducing the complexity of the actual AMC system and making the classification accuracy close to optimum. The study in [23] gives a deep learning model based on one-dimensional convolution neural network to classify large-scale coded high-dimensional sequences, thus realizing radar emitter classification. A blind modulation classification method of combining time-frequency distribution and CNN is employed in [24]. By processing time-frequency diagram as an image, a series of frequency-modulated signals, including linear frequency-modulated signal, sinusoidal frequency signal, and polynomial frequency signal, are identified and classified. The study in [25] accomplishes feature extraction using short-time Fourier transform and AMC based on support vector machine of various types signals, such as single frequency, liner frequency modulation, binary frequency shift keying, 4-frequency shift keying, BPSK, and 16QAM. The superiority of the proposed algorithm lies in that it can classify different modulation types, not just for one specific type. A method based on CNN is carried out to discriminate seven types of pulse repetitive interval modulation in [26], which is robust to the environment with lost and spurious pulses.

From the above literature, it can be seen that using deep learning to achieve AMC of signals is a hot research topic. As a new research direction of machine learning, deep learning $[27,28]$ has made breakthroughs in many fields in recent years. Deep learning achieves complex nonlinear function approximation, which has stronger generalization ability, and depicts more abundant essential information of the data samples compared with the conventional shallow model. Due to its excellent performance, deep learning is widely applied in computer vision [29], speech recognition [30], image recognition [31], radar target recognition [32], classification of radar and communication signals in electronic warfare systems [33], and so on. Accordingly, on the basis of the complex jamming environment faced by cognitive radar, this paper utilizes the deep learning technology based on $\mathrm{CNN}$ to carry out the classification of jamming modulation automatically, realizing jamming environment perception for cognitive radar.

Besides cognitive radar, modulation classification has been considered in cognitive radio systems, for military application in communication systems, and more frequently in optical communication systems $[34,35]$. The study of this paper can be extended to modulation classification in these areas [36].

The rest of this paper is organized as follows. Section 2 introduces the principle of AMCOJ based on CNN for cognitive radar. Section 3 details the multidimensional feature map construction method for seven types of jamming. We design the CNN network structure in Section 4. Section 5 verifies the performance of the proposed classification algorithm through computer simulation. The conclusion is drawn in Section 6.

\section{Principle of AMCOJ}

The AMCOJ for a cognitive radar consists of multidimensional feature map construction and CNN design. This paper mainly aims at the AMC of seven types of common jamming, namely, noise frequency modulation jamming (NFMJ), noise amplitude modulation jamming (NAMJ), monofrequency pulse jamming (MFPJ), noise frequencymodulated pulse jamming (NFMPJ), dense repeater jamming (DRJ), sparse repeater jamming (SRJ), and slice jamming (SJ, also known as interrupted-sampling and repeater jamming (ISRJ)). The characteristics of the above interferences are as listed in Table 1. 


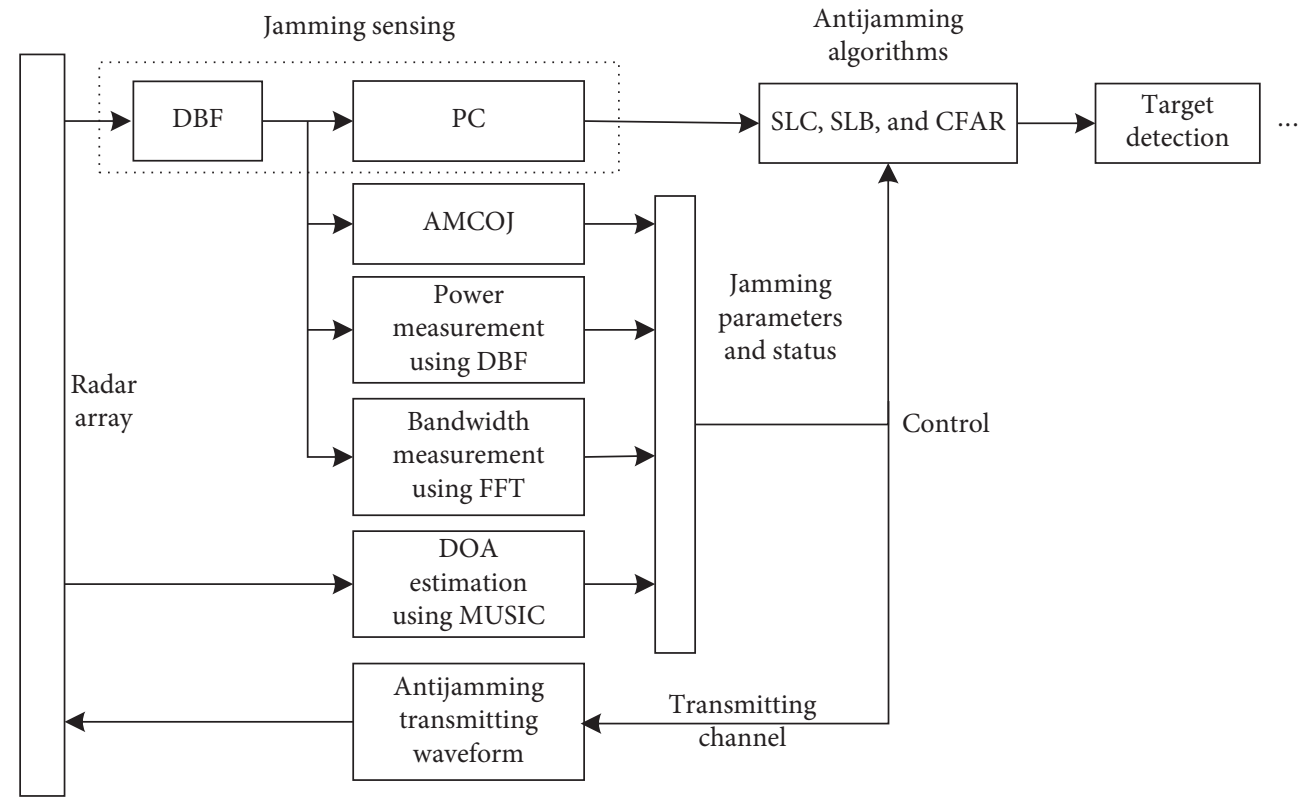

FIGURE 1: The principle of antijamming for a cognitive radar.

TABLE 1: The characteristics of the seven types of jamming.

\begin{tabular}{lc}
\hline Types & Characteristics \\
\hline NFMJ & Noise-modulated frequency and constant envelope \\
NAMJ & Noise-modulated amplitude \\
MFPJ & Pulses modulated in single frequency \\
NFMPJ & Random pulse width and noise-modulated frequency \\
DRJ & Repeat back radar signal with pulse repetition interval less than the transmitted pulse width of the radar \\
SRJ & Repeat back radar signal with pulse repetition interval greater than the transmitted pulse width of the radar \\
SJ & Repeat back radar signal in cutting segments \\
\hline
\end{tabular}

Figure 2 shows the diagram of the jamming sensing network in a cognitive radar. The jamming to a cognitive radar can be roughly divided into two categories, coherent or noncoherent. Since it is coherent to the radar, the waveforms of the repeater jamming before and after pulse compression change significantly. Hence, the data before and after PC processing is selected as the input of the jamming sensing network as shown in Figure 2.

This paper mainly focuses on the radar environment perception processing consisting of multidimensional feature map construction and CNN model design. The differences of different jamming types can be observed in time and frequency domain, so that maps of short-time Fourier transform (STFT) and envelopes before and after PC can be used as features. This paper proposes the method of constructing multidimensional feature map to ensure the correct recognition rate, since single feature map is not effective in discriminating some jamming types. For instance, it is difficult to discriminate noise frequency modulation jamming from noise amplitude modulation jamming in the time-frequency domain. However, there is an obvious difference between them in the time domain before PC. As shown in Figure 2, the feature of time frequency and envelopes can be obtained by STFT analysis and envelope calculation, respectively. The one-dimensional envelope before and after PC can be graphically processed in the time domain to form a two-dimensional image suitable for the use of CNN. The STFT result can be transformed to be a twodimensional feature map by threshold quantization. The three feature maps are merged to form a multidimensional feature map. The CNN adopting the classical 2-dimensional convolution network structure treats the two-dimensional data of the multidimensional feature map as its input and employs the convolutional kernel to synthetically perceive the input. Through training, the network is continuously updated to its optimum and finally, the seven classes of jamming are recognized.

\section{Construction of the Multidimensional Feature Map}

Deep learning based on CNN is now widely employed in the field of image recognition. If we transform the jamming features into images in a two-dimensional map, $\mathrm{CNN}$ will be possible to be applied to extract their differences as its recognition process of an image. In order to improve the classification accuracy of the jamming using $\mathrm{CNN}$ approach, this paper proposes the idea of extracting multiple jamming features from a multidimensional feature map. Envelope features and time-frequency spectrum features are selected 


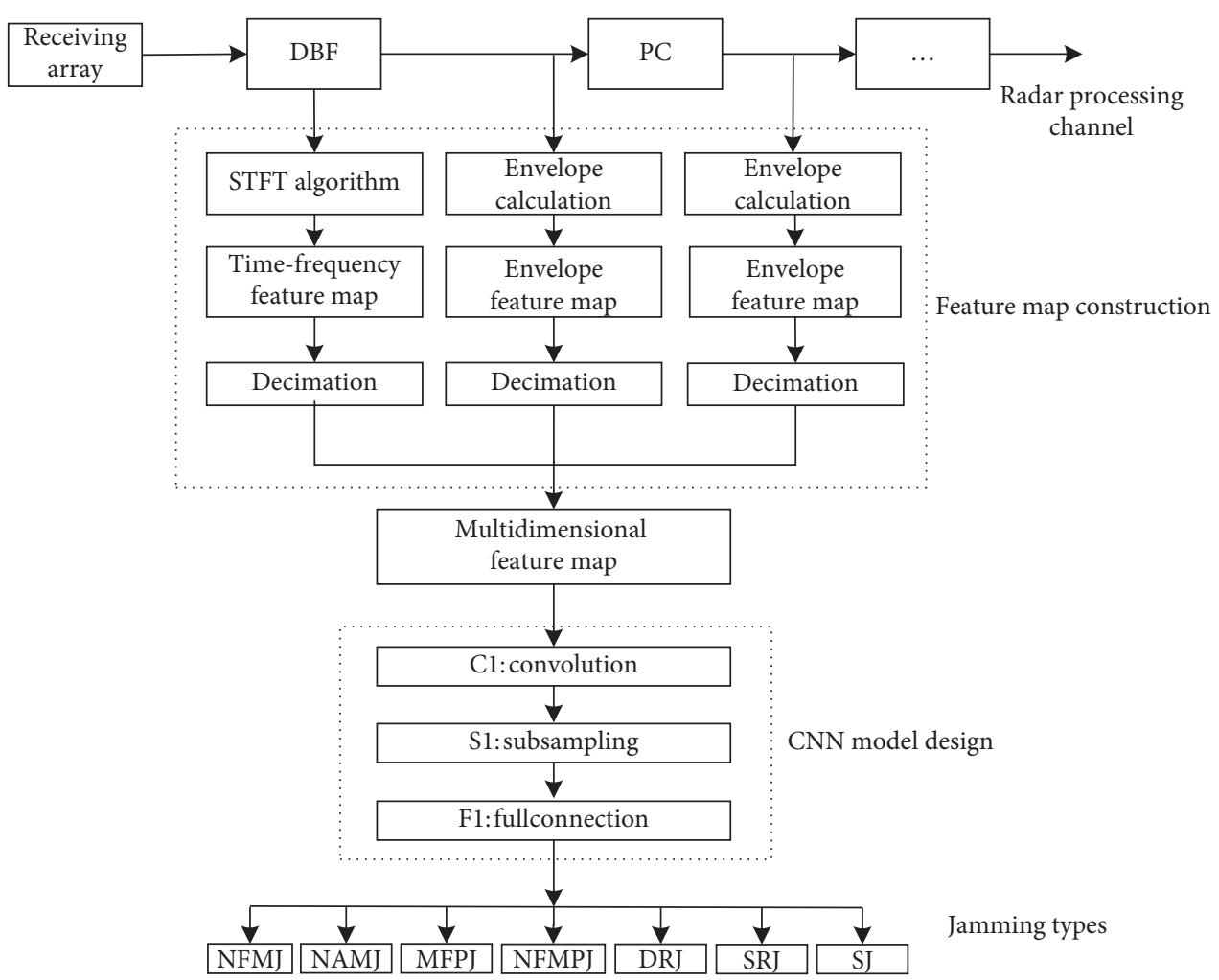

FIgURE 2: The principle diagram of AMCOJ in a cognitive radar.

to form the feature map. The one-dimensional envelope features before and after pulse compression are plotted in the time domain as planar maps, and the time-frequency features are quantified in amplitude and turn into a two-dimensional planar map, either. Then the above planar maps are combined to form a multidimensional feature map used as the input of $\mathrm{CNN}$. The construction process of each feature map is discussed as follows.

3.1. Time-Frequency Feature Map. Obvious differences can be observed in the time-frequency map of the interferences. The short-time Fourier transform (STFT) can be used to obtain the time-frequency spectrum and can be written as

$$
S(m, k)=\sum_{n=0}^{N_{s}-1} s(n) h(n-m) e^{-j\left(2 \pi / N_{f}\right) n k}, \quad k=0,1,2, \ldots, N_{f}-1,
$$

where $n, k, N_{s}, N_{f}$, and $m$ represent the discrete-time instant, the discrete frequency, the sample number, the total frequency number, and time delay, respectively, and $h(n-$ $m)$ is the rectangular window function. Formula (1) indicates that $S(m, k)$ is 2-dimensional data and can be transformed into a time-frequency feature map $o_{1}$ after quantization.

The preprocessing of $|S(m, k)|(|\cdot|$ denotes the absolute value) mainly includes image binarization and geometric transformation. Image binarization refers to setting the gray value of the pixels on the image to 0 or 1 by comparing the values of the map with a threshold. Geometric transformation means that the scaling of an image is transformed into a desired size. Taking slice jamming as an example, the time-frequency spectrum gained by STFT is shown in Figure 3(a). The absolute value of STFT of slice jamming is a two-dimensional matrix data. By setting threshold $\rho_{1}$ (which is set to the half of the peak value), the value larger than $\rho_{1}$ in the matrix is quantized to 1 and vice versa to 0 . The binary feature map of slice jamming is shown in Figure 3(b). The analysis of the other types of radar jamming is similar to that of slice jamming, and the binary feature maps are shown in Figures 4(a)-4(f), respectively. Similar to that of noise frequency modulation jamming in Figure 3(a), the binary feature map of noise amplitude modulation jamming is disordered as shown in Figure 3(b). One frequency can be observed in the monofrequency pulse jamming map in Figure 4(c). Since noise frequency modulation is used to modulate the pulse, the frequency variation of noise frequency-modulated pulse jamming is irregular in Figure 4(d). Figures $4(\mathrm{e})$ and $4(\mathrm{f})$ show that the distribution of binary feature maps of dense repeater jamming and sparse repeater jamming is still similar to the transmitted linear frequency modulation signal. Therefore, we can conclude that it is difficult to distinguish between noise frequency modulation jamming, noise amplitude modulation jamming, and noise frequency-modulated pulse jamming according to the timefrequency feature alone.

3.2. Envelope Feature Map before PC. According to the characteristics of the seven classes of jamming in the time domain, the envelope features before PC can be extracted. The data before PC (after DBF) is a one-dimensional vector; 


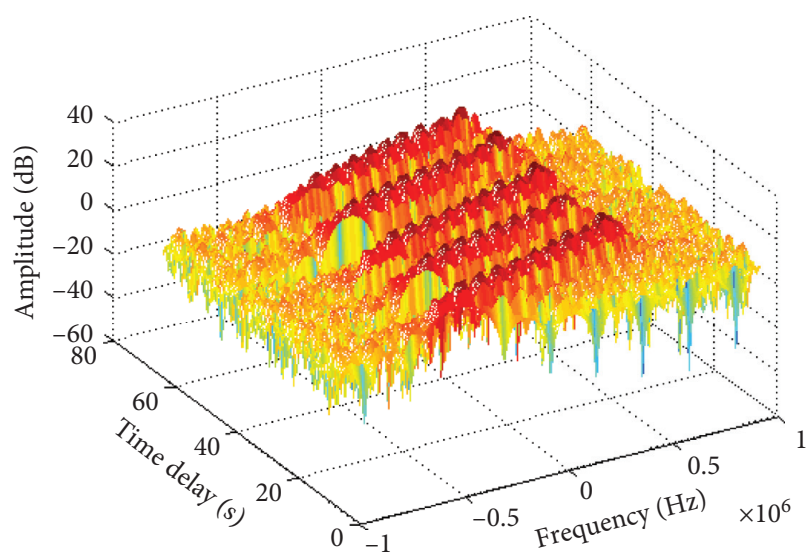

(a)

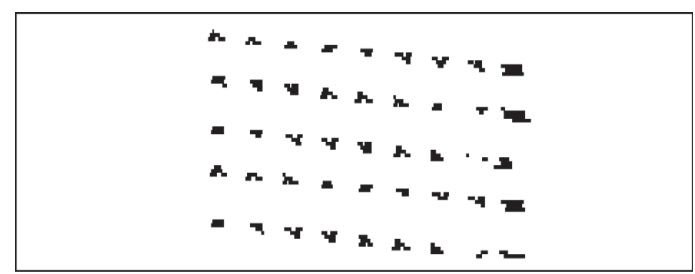

(b)

Figure 3: The time-frequency feature of SJ. (a) Time-frequency spectrum of SJ. (b) Binary time-frequency feature map of SJ.

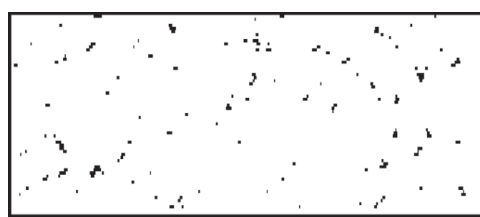

(a)

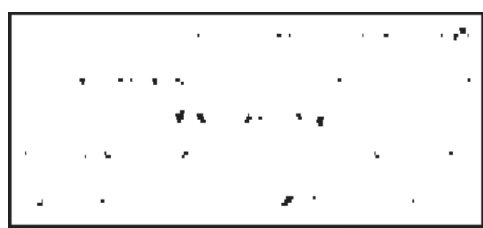

(d)

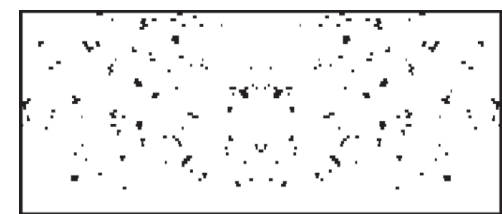

(b)

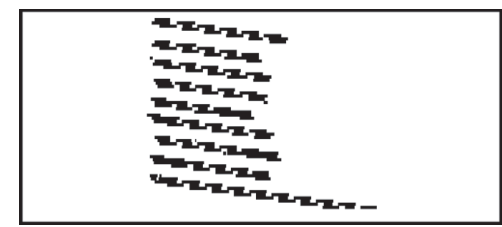

(e)

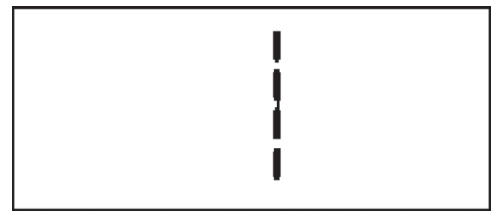

(c)

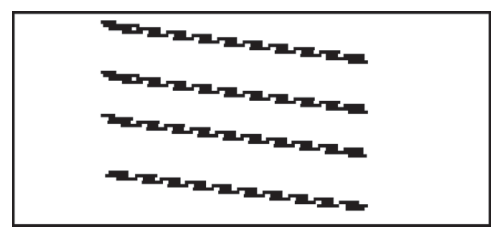

(f)

Figure 4: Binary time-frequency feature maps of different types of jamming. (a) NFMJ. (b) NAMJ. (c) MFPJ. (d) NFMPJ. (e) DRJ. (f) SRJ.

it can be depicted as a binary map by drawing the envelope in the time domain. The envelope feature map before PC is termed as $\mathrm{O}_{2}$. We establish a 2-dimensional decision matrix latticed by rows and columns according to the required map size and judge whether there is a plot falling within the corresponding grids. If there is a point falling in the grids, the pixel in the envelope map is recorded as 1. Otherwise, it is recorded as 0 . The schematic diagram of the decision matrix is shown in Figure 5. Taking slice jamming as an example, the envelope of slice jamming before PC (after DBF) process is shown in Figure 6(a). By means of the graphical processing, the envelope feature map is illustrated in Figure 6(b). Similarly, the creation of the remaining jamming maps is similar to slice jamming and can be observed in Figure 7. Due to the similarity of monofrequency pulse jamming, noise frequency-modulated pulse jamming, and sparse repeater jamming in the envelope characteristics, we have trouble to distinguish between these three types of jamming barely according to this feature.

3.3. Envelope Feature Map after PC. We further extract the envelope feature after PC. Since the data processed by PC is a one-dimensional vector, it is essential to form a binary map $o_{3}$ by the above graphical processing. Still taking slice jamming as an example, the waveform and binary feature map after PC are shown in Figures 8(a) and 8(b), respectively. Similarly, the map construction of the remaining six types of jamming is the same and can be observed in Figure 7. However, the similarity of noise frequency modulation jamming and noise amplitude modulation jamming in the envelope feature makes it difficult to discriminate between the two.

3.4. Multidimensional Feature Map. Viewed from the timefrequency feature map, noise frequency modulation jamming, noise amplitude modulation jamming, and noise frequency-modulated pulse jamming are not easy to be distinguished. From the perspective of the envelope before PC, monofrequency pulse jamming, noise frequencymodulated pulse jamming, and sparse repeater jamming are prone to be confused and indistinguishable. Through the envelope feature map after PC, it is difficult to differentiate between noise frequency modulation jamming and noise amplitude modulation jamming. The schematic diagram of multidimensional feature map $o_{4}$, as shown in Figure 9, is constructed by combining the above three single feature 


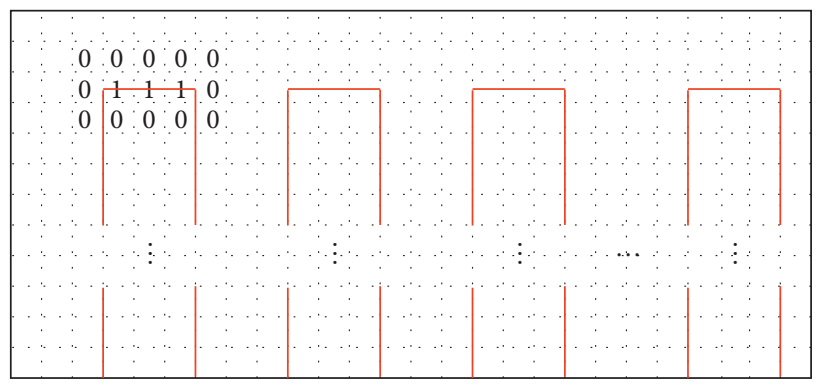

$64 \times 512$ lattices

Figure 5: The schematic diagram of decision matrix.

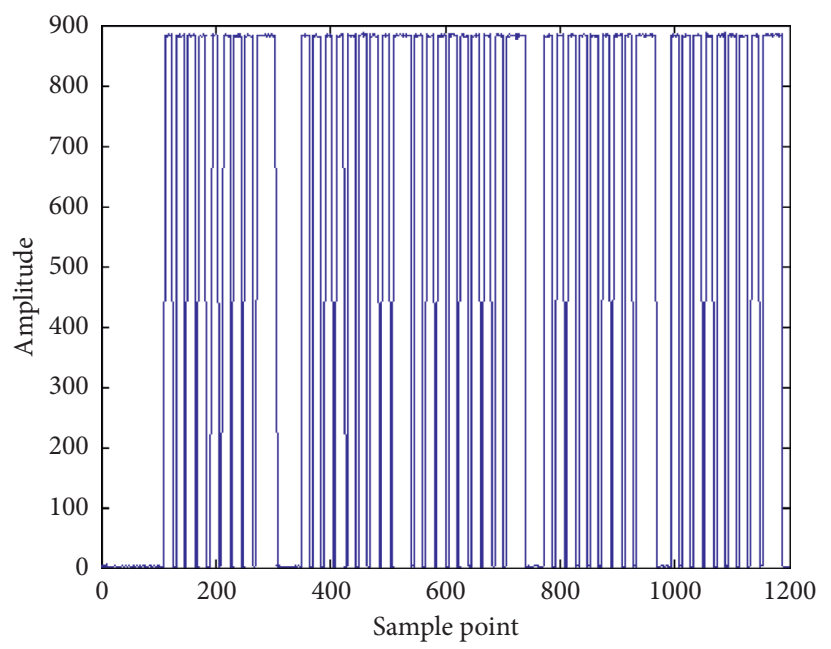

(a)

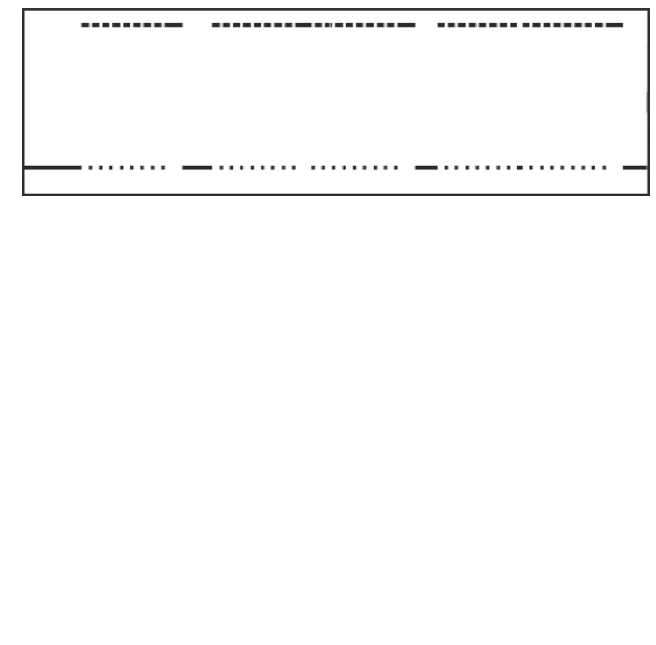

(b)

Figure 6: The envelope feature of SJ before PC. (a) The envelope of SJ before PC. (b) The corresponding binary envelope feature map of SJ.

maps, which mark the upper part as "time frequency," middle part as "envelope before PC," and bottom part as "envelope after PC." The multidimensional feature map of the seven classes of jamming is observed in Figure 7, it can be seen that noise frequency modulation jamming, noise amplitude modulation jamming, and noise frequency-modulated pulse jamming can be distinguished according to the envelope feature map before $\mathrm{PC}$, and monofrequency pulse jamming, noise frequency-modulated pulse jamming, and sparse repeater jamming are apt to discriminate through the time-frequency feature map. Therefore, seven classes of jamming can be classified simultaneously using the multidimensional feature map.

\section{Design and Analysis of the CNN Model}

The architecture of a typical CNN is generally composed of five stages: input layer, convolution layer, sampling layer, full connection layer, and output layer [37]. We propose a 2dimensional CNN model according to the complexity of our data. Without using the conventional local field perception in many $\mathrm{CNN}$ applications, we design convolution kernels to synthetically perceive the features in the three maps simultaneously. As shown in Figure 9, the network consists of one input layer, one convolution layer, one max-pooling layer, one fully connected layer, and one output layer. The first layer is the input layer, and its inputs are the three feature maps with dimensions of $64 \times 512$. The second layer is the convolution layer $\mathrm{C} 1$ with six convolution kernels of size $192 \times 1$. Considering the dimension of input feature data, the linear convolution kernel extracts the multiple feature information synthetically from the three feature maps. After the process in this layer, the 2-dimensional feature map is transformed into 61 -dimensional feature maps with the size of $1 \times 512$. The third layer S1 is the sampling layer, compressing the feature map into $1 \times 64$ by max-pooling with the size of $1 \times 8$ and reducing the complexity of the model. The fourth layer F1 is the full connection layer. Since the feature map generated is 1dimensional, the feature maps can be connected successively to form a $384 \times 1$ feature vector. Finally, the output layer maps the feature data to 0 and 1 by a sigmoid function and outputs the corresponding maximum probability value as the jamming class.

The training process of CNN mainly includes two stages: forward propagation and backward feedback. Firstly, the training samples carry out forward propagation network according to the flow of Figure 10. Secondly, comparing the errors between the output and the expectation values, the parameter sensitivity is calculated to update the weights and 


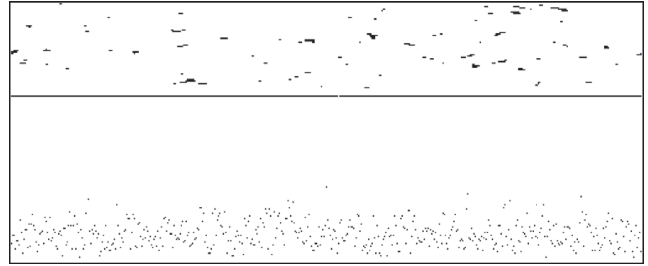

(a)

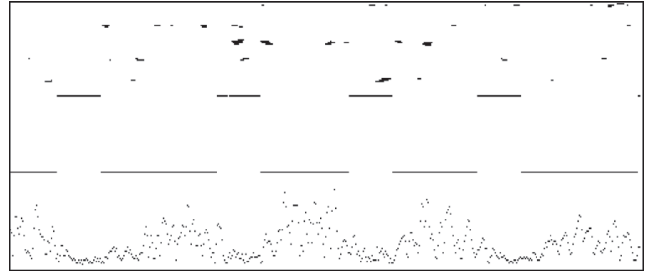

(c)

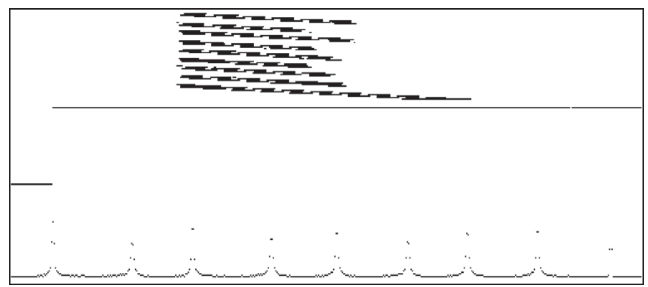

(e)

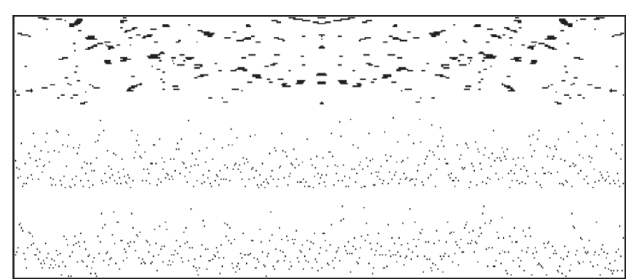

(b)

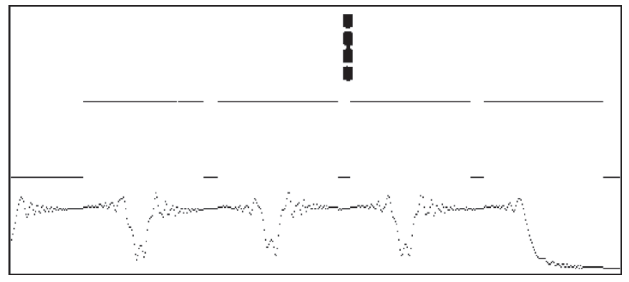

(d)

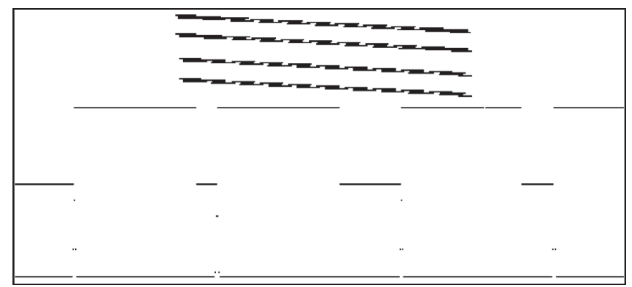

(f)

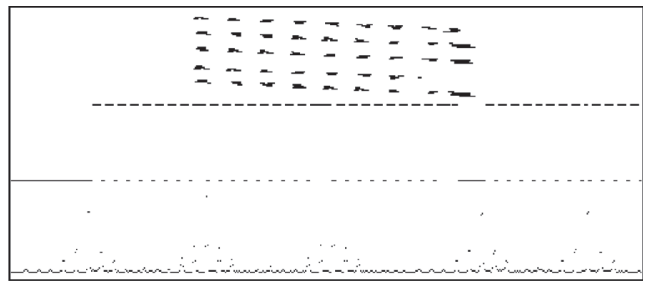

$(\mathrm{g})$

FiguRE 7: The multidimensional feature map of the seven classes of jamming. (a) NFMJ. (b) NAMJ. (c) MFPJ. (d) NFMPJ. (e) DRJ. (f) SRJ. (g) SJ.

biases of each layer, that is, to enter the backward feedback network. By constantly updating weights and biases, the network errors decrease as the cost function converges, and the training of the network model is completed. In the testing phase, the weights and biases trained in the training phase are used to classify the test data.

\section{Numerical Simulations}

5.1. Parameter Setting. In this section, we present a variety of simulation experiments to demonstrate the performance of our proposed AMCOJ algorithm using CNN based on multidimensional feature map (MD-CNN). Seven different modulation candidates are considered here, namely, noise frequency modulation jamming, noise amplitude modulation jamming, monofrequency pulse jamming, noise frequency-modulated pulse jamming, dense repeater jamming, sparse repeater jamming, and slice jamming. Since the robustness of the MD-CNN algorithm is deeply affected by the training data samples, training samples in different conditions should be included in the sample database. Hence, we generate the training samples via changing the jamming principal parameters such as time delay, slice period, and repeat interval. The jamming parameter settings are shown in Table 2. Linear frequency modulation signal is adopted as the radar transmitting signal, the bandwidth of which varies from $4 \mathrm{kHz}$ to $1 \mathrm{MHz}$ and the pulse width is from $40 \mu$ s to $120 \mu$ s. In addition, the pulse repetition period of the transmitted signal is set as $600 \mu$ s and sampling frequency is $2 \mathrm{MHz}$. The jamming-to-noise ratio (JNR) ranges from 3 to $15 \mathrm{~dB}$ subject to the additive white Gaussian noise.

In the network training stage, the feature maps are gained by the processing of the envelope and time-frequency spectrum. We merge three feature maps with the size of $64 \times 512$ separately into one $192 \times 512$ multidimensional feature map and use it as the sample input for CNN. The iteration number of $\mathrm{CNN}$ is set to 50 . The network model is trained by 350 samples and tested by another 175 samples.

5.2. Performance Analysis. The correct recognition rates of the seven jamming classes using MD-CNN algorithm under different JNRs are illustrated in Table 3. Errors are prone to 


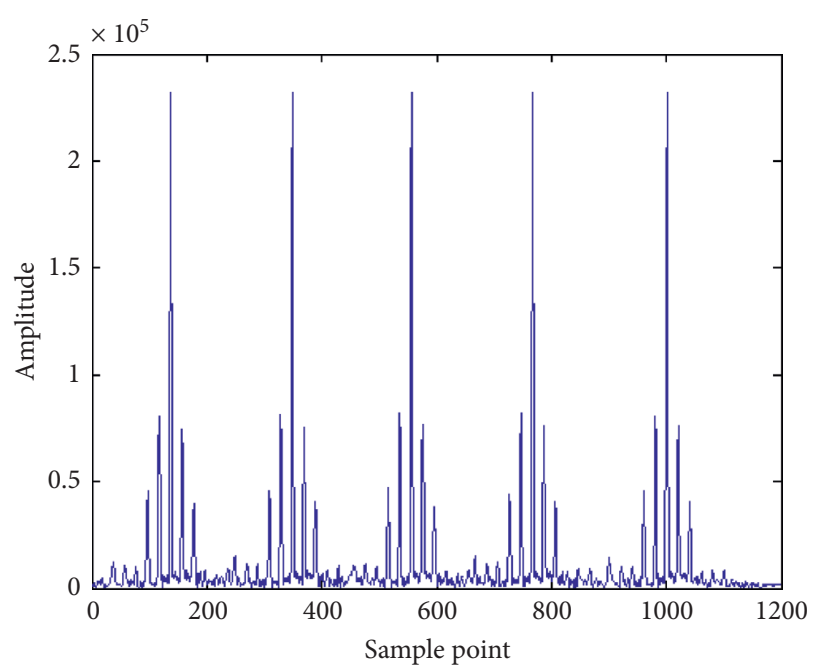

(a)

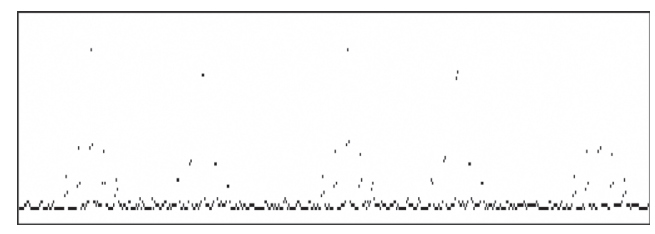

(b)

FIgURe 8: The envelope feature of SJ after PC. (a) The envelope after PC. (b) The corresponding binary envelope feature map.

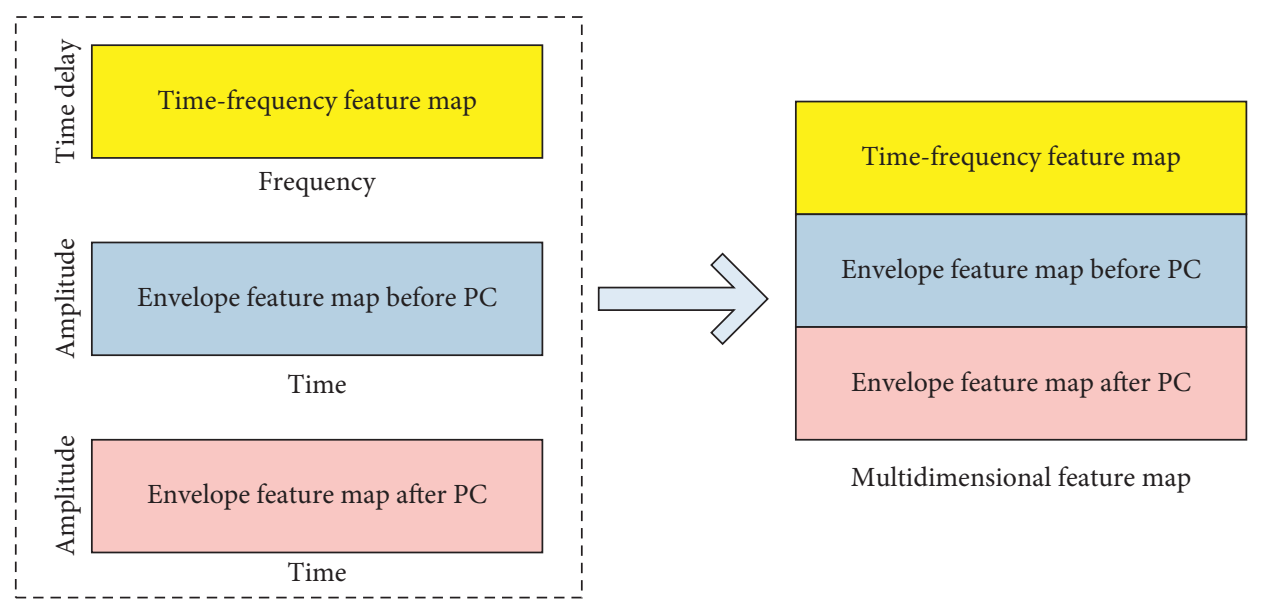

Figure 9: The schematic diagram of multidimensional feature map.

occur under low JNR circumstances due to the high similarity between noise frequency-modulated pulse jamming and noise amplitude modulation jamming in the time domain and time-frequency domain. It can be seen from the data in Table 3 that the numbers of mispredicted samples of each jamming degrade with the increase of JNR.

We compare the performance of the proposed method with the single feature map method using STFT feature map and envelope feature map before and after PC. The total correct recognition rates based on the single feature maps and the multidimensional feature map are plotted in Figure 11. Under the same JNR conditions, the classification accuracy of our proposed algorithm is higher than that of the single feature map algorithm. The result demonstrates that the correct recognition rate of the jamming is effectively improved by constructing the multidimensional feature map. In addition, under the scenario of $\mathrm{JNR}=8 \mathrm{~dB}$ and above, the correct recognition rate of our proposed method reaches over 95\%. The results indicate that the proposed algorithm is effective in discriminating the modulated jamming candidates.

\subsection{Comparison Study with Different Convolutional Kernels and Feature Extraction Approach}

5.3.1. Comparison of CNN Model with Different Convolutional Kernels. The selection of the CNN kernel is of great significance to the CNN performance. As shown in Figure 12, the CNN algorithms with several types of convolution kernel are simulated as a comparison to our proposed $\mathrm{CNN}$ with linear kernel. Under the scenario of JNR $=15 \mathrm{~dB}$, the parameter setting and correct recognition rates of the CNN models with different convolution kernels are shown in Table 4. As can be seen from Table 4, CNN1, CNN2, and CNN3 with convolution kernel of 1-channel $192 \times n 1$ 


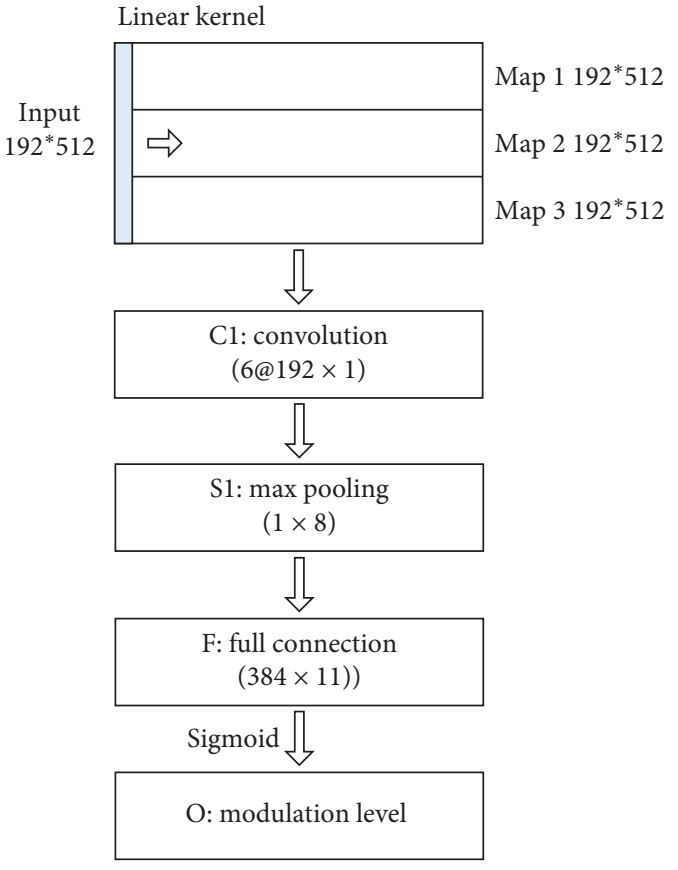

Figure 10: Diagram of the CNN model.

TABle 2: Parameter setting of different types of jamming.

\begin{tabular}{|c|c|}
\hline Type & Parameter setting \\
\hline NFMJ & Noise-modulated frequency \\
\hline NAMJ & Noise-modulated amplitude \\
\hline MFPJ & $\begin{array}{l}\text { The carrier frequency: } 10 \mathrm{kHz}-100 \mathrm{kHz} \text {; } \\
\text { pulse width: } 40 \mu \mathrm{s}-120 \mu \mathrm{s}\end{array}$ \\
\hline NFMPJ & $\begin{array}{l}\text { Pulse repetition period: random in } 140-250 \text {; } \\
\text { pulse width (sample point): random in } 60-120\end{array}$ \\
\hline DRJ & $\begin{array}{l}\text { Repeat interval (sample point): random value }(50-100) \text {, } \\
\text { less than the transmitted pulse width }\end{array}$ \\
\hline SRJ & $\begin{array}{l}\text { Repeat interval (sample point): random value }(180-250) \text {, } \\
\text { greater than the transmitted pulse width }\end{array}$ \\
\hline SJ & $\begin{array}{l}\text { The slice period (sample point): } 20 \text {; } \\
\text { slice length (sample point): } 1-19 ; \\
\text { time delay (sample point): random in } 140-250\end{array}$ \\
\hline
\end{tabular}

dimension have a better performance than those of CNN4 and CNN5, which means that multifeature comprehensive perception of the three maps simultaneously is superior to local perception of them in succession.

The same conclusion can be drawn that the performance of CNN6, CNN7, and CNN8 with three channels sensing the three feature maps degrades slightly in comparison with CNNs1-3. We take the CNN6 model with three channels as an example to analyze the numbers of mispredicted samples of each jamming class in detail, as shown in Table 5. Compared with the data in Table 3, we can see that the performance of CNN1 with one channel is superior to that of CNN6 with three channels for most of the jamming classes.

5.3.2. Comparison Study of the Feature Extraction Approach Using Radar Ambiguity Function. As a conventional approach, radar ambiguity function has been utilized to extract the modulation type of the jamming feature. Here, as a comparison study, we use the combination of radar ambiguity function and a type of classical CNN called LeNet5 (termed as RA+LeNet5) to classify different jamming signals.

LeNet5 here used is composed of 7 layers: (1) the first convolution layer with $5 \times 5$ kernels; (2) the first pooling layer with $62 \times 2$ kernels; (3) the second convolution layer with $165 \times 5$ kernels; (4) the second pooling layer with 16 $5 \times 5$ kernels; (5) the third convolution layer with $1205 \times 5$ kernels; (6) full connection layer with 120 output; (7) output layer with 7 types of output.

We test the performance of RA + LeNet5 and our proposed method of CNN1 in Table 4 with 350 samples after a training process of 700 samples. The numbers of mispredicted samples of each jamming class are illustrated in Table 6. Fewer mistakes can be observed with CNN1 of this paper at JNR of $6 \mathrm{~dB}$ and $8 \mathrm{~dB}$, respectively. As we have pointed out, the superior performance of our proposed CNN1 lies mainly in that it can perceive three features in three maps simultaneously.

The algorithm complexity of RA + LeNet5 and the method of this paper can be analyzed in two aspects, the feature extraction phase and the classification phase.

Algorithm complexity in feature extraction map construction phase is as follows:

(1) The method of this paper: there are 64-point FFT of 30 times to calculate the short Fourier transform and $2 \mathrm{~N}$ multiplications for amplitude calculation without rooting operation, where $N$ refers to the data length

(2) RA + LeNet5: to calculate the radar ambiguity function, there is a 64-point FFT of 30 times in the 
TABLE 3: Numbers of mispredicted samples of MD-CNN at different JNRs.

\begin{tabular}{lcccccc}
\hline Type & $\mathrm{JNR}=3 \mathrm{~dB}$ & $\mathrm{JNR}=5 \mathrm{~dB}$ & $\mathrm{JNR}=8 \mathrm{~dB}$ & $\mathrm{JNR}=10 \mathrm{~dB}$ & $\mathrm{JNR}=13 \mathrm{~dB}$ & $\mathrm{JNR}=15 \mathrm{~dB}$ \\
\hline NFMJ & 3 & 0 & 0 & 0 & 0 & 0 \\
NAMJ & 0 & 0 & 0 & 0 & 0 & 0 \\
MFPJ & 0 & 0 & 0 & 1 & 0 & 0 \\
NFMPJ & 7 & 5 & 0 & 0 & 0 & 0 \\
DRJ & 1 & 0 & 0 & 0 & 0 \\
SRJ & 1 & 4 & 1 & 0 & 0 \\
SJ & 5 & & & 0 & 0 \\
\hline
\end{tabular}

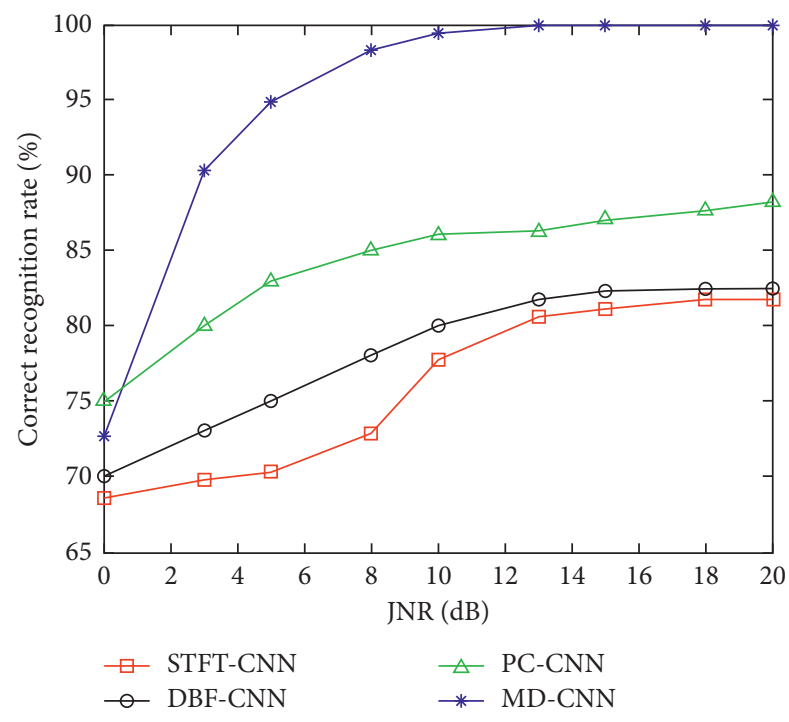

FigURE 11: Correct recognition rates for different algorithms.

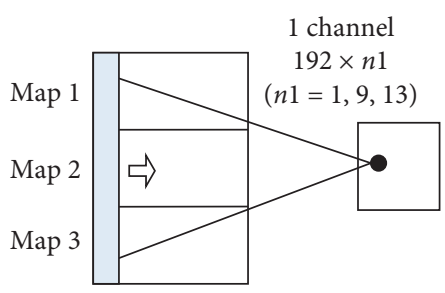

CNNs $1-3$

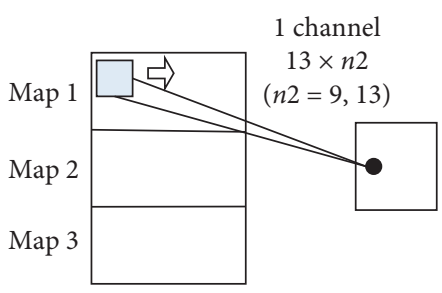

CNNs 4-5

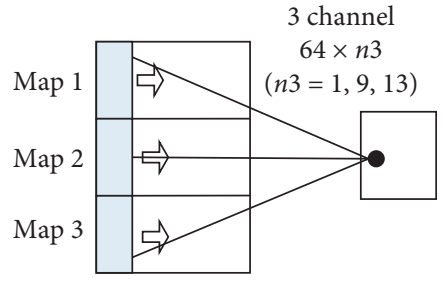

CNNs 6-8

FIGURE 12: CNNs model with different kernel structures.

TABLE 4: Performance comparisons between CNNs with different kernel structures.

\begin{tabular}{lccc}
\hline Model & Channel number & Convolution kernel sizes in each channel & Correct recognition rates (\%) \\
\hline CNN1 (linear kernel) & 1 & $192 \times 1$ & 100 \\
CNN2 & 1 & $192 \times 9$ & 98.28 \\
CNN3 & 1 & $192 \times 13$ & 99.42 \\
CNN4 & 1 & $13 \times 13$ & 14.28 \\
CNN5 & 1 & $13 \times 9$ & 14.28 \\
CNN6 & 3 & $64 \times 1$ & 92.57 \\
CNN7 & 3 & $64 \times 9$ & 82.86 \\
CNN8 & 3 & $64 \times 13$ & 90.86 \\
\hline
\end{tabular}


TABLE 5: Numbers of mispredicted samples of CNN6 at different JNRs.

\begin{tabular}{|c|c|c|c|c|c|c|}
\hline Type & $\mathrm{JNR}=3 \mathrm{~dB}$ & $\mathrm{JNR}=5 \mathrm{~dB}$ & $\mathrm{JNR}=8 \mathrm{~dB}$ & $\mathrm{JNR}=10 \mathrm{~dB}$ & $\mathrm{JNR}=13 \mathrm{~dB}$ & $\mathrm{JNR}=15 \mathrm{~dB}$ \\
\hline NFMJ & 1 & 0 & 0 & 0 & 0 & 0 \\
\hline NAMJ & 2 & 0 & 0 & 0 & 0 & 0 \\
\hline MFPJ & 5 & 5 & 1 & 0 & 0 & 0 \\
\hline NFMPJ & 5 & 5 & 2 & 1 & 1 & 1 \\
\hline DRJ & 9 & 4 & 2 & 1 & 0 & 0 \\
\hline SRJ & 11 & 10 & 10 & 10 & 9 & 8 \\
\hline SJ & 17 & 12 & 11 & 8 & 7 & 4 \\
\hline
\end{tabular}

TABLE 6: Numbers of mispredicted samples of RA + LeNet5 and the method of this paper.

\begin{tabular}{lcccc}
\hline JNR & \multicolumn{2}{c}{$6 \mathrm{~dB}$} & \multicolumn{2}{c}{$8 \mathrm{~dB}$} \\
Method & $\begin{array}{c}\text { CNN1 of } \\
\text { this paper }\end{array}$ & RA + LeNet5 & $\begin{array}{c}\text { CNN1 of } \\
\text { this paper }\end{array}$ & RA + LeNet5 \\
\hline NFMJ & 0 & 5 & 0 & 3 \\
NAMJ & 0 & 19 & 0 & 15 \\
MFPJ & 0 & 22 & 0 & 14 \\
NFMPJ & 0 & 23 & 0 & 14 \\
DRJ & 3 & 25 & 1 & 11 \\
SRJ & 8 & 22 & 6 & 16 \\
SJ & 10 & 16 & 6 & 17
\end{tabular}

time delay domain and 64-point FFT of 30 times in the Doppler domain

The number of convolutions is used to compare the algorithm complexity in the classification phase, since convolutions can represent the main complexity for these phases:

(1) Classification method of this paper: convolution kernel of $192 \times 1$

(2) RA + LeNet5: $65 \times 5$ convolution kernels $+165 \times 5$ convolution kernels $+1205 \times 5$ convolution kernels

We can conclude from this comparison that, in the feature extraction map construction phase, the complexity of the two methods is the same, while in the classification phase, the multikernel and multiconvolution layer structure makes RA + LeNet5 more complex than that of our method from the viewpoint of the convolution numbers.

\section{Conclusion}

We employ the CNN approach to solve the recognition of jamming for cognitive radar. Considering the differences between different types of jamming in envelope and timefrequency spectrum, the multidimensional feature map is constructed by combining three features of the received signal including amplitude before and after PC and STFT analysis. After the training process, the CNN observes the local features on the combined map and gives the classification result automatically. Computer simulation reveals that the sensing and classification of the jamming classes based on the multidimensional feature map achieve superior performance in comparison with the single feature map method. In addition, multifeature comprehensive perception of the three pictures simultaneously with a linear kernel $\mathrm{CNN}$ is superior to $\mathrm{CNN}$ with other kernel types.

\section{Data Availability}

The data used to support the findings of this study are from the simulation conditions of open literature, and the authors made computer simulations to generate all the simulation data according to these conditions.

\section{Conflicts of Interest}

The authors declare that they have no conflicts of interest.

\section{Acknowledgments}

This work was partially supported by the Foundation of Science and Technology on Electronic Information Control Laboratory.

\section{References}

[1] S. Haykin, "Cognitive radar: a way of the future," IEEE Signal Processing Magazine, vol. 23, no. 1, pp. 30-40, 2006.

[2] G. Huang and L. Yang, "A radar anti-jamming technology based on blind source separation," Journal of Circuits \& Systems, vol. 3, no. 6, pp. 2021-2024, 2004.

[3] G. Yue and X. Wang, "Anti-jamming coding techniques with application to cognitive radio," IEEE Transactions on Wireless Communications, vol. 8, no. 12, pp. 5996-6007, 2009.

[4] L. Rosenberg and D. Gray, "Anti-jamming techniques for multichannel SAR imaging," IEE Proceedings-Radar, Sonar and Navigation, vol. 153, no. 3, pp. 234-242, 2006.

[5] M. A. Richards, J. A. Scheer, and W. A. Holm, Principles of Modern Radar, SciTech Publishing, Raleigh, NC, USA, 2010.

[6] J. M. Kim, J. C. Ye, and J. C. Ye, "Compressive MUSIC: revisiting the link between compressive sensing and array signal processing," IEEE Transactions on Information Theory, vol. 59, no. 9, pp. 6148-6149, 2013.

[7] L. Zhao, M. Amin, A. Lindsey et al., "Subspace array processing for the suppression of FM jammers in GPS receivers," IEEE Transactions on Aerospace \& Electronics Systems, vol. 40, no. 1, pp. 80-92, 2004.

[8] C. Fernandez-Prades, J. Arribas, and P. Closas, "Robust GNSS receivers by array signal processing: theory and implementation," Proceedings of the IEEE, vol. 104, no. 6, pp. 1-14, 2016. 
[9] F. Yan, M. Jin, and X. Qiao, "Low-complexity DOA estimation based on compressed MUSIC and its performance analysis," IEEE Transactions on Signal Processing, vol. 61, no. 8, pp. 1915-1930, 2013.

[10] H. T. Wu, J. F. Yang, and F. K. Chen, "Source number estimators using transformed Gerschgorin radii," IEEE Transactions on Signal Processing, vol. 43, no. 6, pp. 1325-1333, 1995.

[11] C. Jiang, M. Gao, Y. Shi et al., "A new method for anti-noise FM interference," Wireless Sensor Network, vol. 1, no. 4, pp. 294-299, 2009.

[12] S. W. Chen, "The 2-D cosinusoidal phase modulation repeater jamming of SAR," Journal of Electronics \& Information Technology, vol. 31, no. 8, pp. 1862-1866, 2009.

[13] Y. H. Lee and S. J. Kim, "Median-prefiltering-based robust acquisition of direct-sequence spread-spectrum signals in wide-band pulse jamming," IEEE Transactions on Vehicular Technology, vol. 51, no. 1, pp. 171-179, 2002.

[14] H. X. Huang, Z. T. Huang, and Y. Y. Zhou, "A study on the shift-frequency jamming to SAR," Journal of Astronautics, vol. 27, no. 3, pp. 463-468, 2006.

[15] O. A. Dobre, M. Oner, S. Rajan, and R. Inkol, "Cyclostationarity-based robust algorithms for QAM signal identification," IEEE Communications Letters, vol. 16, no. 1, pp. 12-15, 2012.

[16] G. Wannberg, A. Pellinen-Wannberg, and A. Westman, "An ambiguity-function-based method for analysis of Doppler decompressed radar signals applied to EISCAT measurements of oblique UHF-VHF meteor echoes," Radio Science, vol. 31, no. 3, pp. 497-518, 2016.

[17] D. Zeng, X. Zeng, G. Lu, and B. Tang, "Automatic modulation classification of radar signals using the generalised timefrequency representation of Zhao, Atlas and Marks," IET Radar, Sonar \& Navigation, vol. 5, no. 4, pp. 507-516, 2011.

[18] R. Debnath, N. Takahide, and H. Takahashi, "A decision based one-against-one method for multi-class support vector machine," Pattern Analysis \& Applications, vol. 7, no. 2, pp. 164-175, 2004.

[19] Z. Jiang, J. Wang, Q. Song, and Z. Zhou, "A refined clusteranalysis-based multibaseline phase-unwrapping algorithm," IEEE Geoscience and Remote Sensing Letters, vol. 14, no. 9, pp. 1565-1569, 2017.

[20] Z. Duona, D. Wenrui, Z. Baochang et al., "Automatic modulation classification based on deep learning for unmanned aerial vehicles," Sensors, vol. 18, no. 3, p. 924, 2018.

[21] A. Ali and F. Yangyu, "Automatic modulation classification using deep learning based on sparse autoencoders with nonnegativity constraints," IEEE Signal Processing Letters, vol. 24, no. 11, pp. 1626-1630, 2017.

[22] A. Ali and F. Yangyu, " $k$-sparse autoencoder based automatic modulation classification with low complexity," IEEE Communications Letters, vol. 21, no. 10, pp. 2162-2165, 2017.

[23] S. Jun, X. Guangluan, R. Wenjuan et al., "Radar emitter classification based on unidimensional convolutional neural network," IET Radar, Sonar \& Navigation, vol. 12, no. 8, pp. 862-867, 2018.

[24] J. Zhang, Y. Li, and J. Yin, "Modulation classification method for frequency modulation signals based on the time-frequency distribution and CNN," IET Radar, Sonar \& Navigation, vol. 12, no. 2, pp. 244-249, 2018.

[25] F. Wang, S. Huang, H. Wang, and C. Yang, "Automatic modulation classification exploiting hybrid machine learning network," Mathematical Problems in Engineering, vol. 2018, Article ID 6152010, 14 pages, 2018.
[26] L. Xueqiong, H. Zhitao, W. Fenghua et al., "Towards convolutional neural networks on pulse repetition interval modulation recognition," IEEE Communications Letters, vol. 22, no. 11, pp. 2286-2289, 2018.

[27] Y. Sun, X. Wang, and X. Tang, "Hybrid deep learning for face verification," IEEE Transactions on Pattern Analysis and Machine Intelligence, vol. 38, no. 10, pp. 1997-2009, 2016.

[28] Y. Chen, Z. Lin, X. Zhao, G. Wang, and Y. Gu, "Deep learning-based classification of hyperspectral data," IEEE Journal of Selected Topics in Applied Earth Observations and Remote Sensing, vol. 7, no. 6, pp. 2094-2107, 2014.

[29] S. Nie, M. Zheng, and Q. Ji, "The deep regression bayesian network and its applications: probabilistic deep learning for computer vision," IEEE Signal Processing Magazine, vol. 35, no. 1, pp. 101-111, 2018.

[30] G. Hinton, L. Deng, D. Yu et al., "Deep neural networks for acoustic modeling in speech recognition: the shared views of four research groups," IEEE Signal Processing Magazine, vol. 29, no. 6, pp. 82-97, 2012.

[31] H.-J. Lee and S.-G. Lee, "Arousal-valence recognition using CNN with STFT feature-combined image," Electronics Letters, vol. 54, no. 3, pp. 134-136, 2018.

[32] J. Pei, Y. Huang, W. Huo et al., "SAR automatic target recognition based on multiview deep learning framework," IEEE Transactions on Geoscience \& Remote Sensing, vol. 56, no. 4, pp. 2196-2210, 2017.

[33] T. Mcconaghy, H. Leung, E. Bosse, and V. Varadan, "Classification of audio radar signals using radial basis function neural networks," IEEE Transactions on Instrumentation and Measurement, vol. 52, no. 6, pp. 1771-1779, 2003.

[34] O. Dobre, "Signal identification for emerging intelligent radios: classical problems and new challenges," IEEE Instrumentation \& Measurement Magazine, vol. 18, no. 2, pp. 11-18, 2015.

[35] S. M. Bilal, G. Bosco, Z. Dong, A. P. T. Lau, and C. Lu, "Blind modulation format identification for digital coherent receivers," Optics Express, vol. 23, no. 20, pp. 26769-26778, 2015.

[36] X. Lin, Y. A. Eldemerdash, O. A. Dobre, S. Zhang, and C. Li, "Modulation classification using received signal's amplitude distribution for coherent receivers," IEEE Photonics Technology Letters, vol. 29, no. 21, pp. 1872-1875, 2017.

[37] H.-C. Shin, H. R. Roth, M. Gao et al., "Deep convolutional neural networks for computer-aided detection: CNN architectures, dataset characteristics and transfer learning," IEEE Transactions on Medical Imaging, vol. 35, no. 5, pp. 12851298, 2016. 